\title{
SCR EN EL RIESGO DE SUSCRIPCIÓN DEL SEGURO DE VIDA: MORTALIDAD, LONGEVIDAD Y REASEGURO
}

\author{
PONS CARDELL, $M^{\mathrm{a}}$ ÀNGELS \\ mapons@ub.edu \\ Universitat de Barcelona, Departamento de Matemática Económica, Financiera y Actuarial \\ Avenida Diagonal 690 (08034) Barcelona \\ SARRASÍ VIZCARRA, F. JAVIER \\ sarrasi@ub.edu \\ Universitat de Barcelona, Departamento de Matemática Económica, Financiera y Actuarial \\ Avenida Diagonal 690 (08034) Barcelona \\ Recibido (28/10/2020) \\ Revisado $(22 / 12 / 2020)$ \\ Aceptado (28/12/2020)
}

RESUMEN: En este trabajo se propone un modelo interno basado en la simulación de Monte Carlo, para el cálculo del capital de solvencia obligatorio del riesgo de suscripción de vida, de una compañía de seguros que presenta dos riesgos, el de supervivencia y el de mortalidad. A diferencia del modelo estándar, la agregación de estos dos riesgos se llevará a cabo sin necesidad de conocer las matrices de correlación. Posteriormente se analiza el efecto que tienen las diferentes modalidades de reaseguro en el capital de solvencia obligatorio. Las modalidades de reaseguro objeto de análisis son el cuota parte, el de excedentes y el stop-loss.

Palabras clave: Reaseguro; Solvencia II; Capital de solvencia obligatorio; mortalidad; longevidad.

ABSTRACT: The aim of this work is to propose an internal model based on the Monte Carlo's simulation method, for the calculation of the solvency capital requirement of the subscription of life's risk, of an insurances company that presents two risks, the survival and mortality ones. Unlike the standard model, the aggregation of these two risks will carry out without knowing the correlation matrixes. Afterwards, the effect that the different modalities of reinsurance have in the solvency capital requirement will be analyzed. The modalities of reinsurance object of analysis are the quota share, the surplus and the stop-loss.

Keywords: Reinsurance; Solvency II; Solvency Capital Requirement; Mortality; Longevity. 


\section{Introducción}

La Directiva de Solvencia II, en el artículo 101, propone calcular el capital de solvencia obligatorio, SCR (Solvency Capital Requirement), mediante un sistema modular basado en los diferentes riesgos que tiene la compañía. En el modelo estándar, el cálculo del $S C R$ se obtiene por agregación de los capitales de solvencia obligatorios de cada uno de los riesgos que integran la cartera. Al tratarse de riesgos dependientes, la agregación no es aditiva, sino que se realiza a través de una suma correlacionada a partir de unas matrices de correlación, cuyos elementos, establecidos por la propia normativa de Solvencia II, recogen los coeficientes de correlación lineal entre los diferentes riesgos.

Existen diferentes técnicas para agregar variables aleatorias, entre ellas cabe destacar:

- Agregación a través de matrices de correlación. Esta es la técnica que se propone en el modelo estándar de Solvencia II y se basa, principalmente, en las hipótesis de normalidad y esperanza cero de las variables aleatorias marginales que se pretenden agregar y que la dependencia de éstas queda totalmente explicada por los coeficientes de correlación lineal.

- Agregación a través de cópulas. Este enfoque permite relajar la hipótesis de normalidad de las variables aleatorias que se agregan a otras funciones de distribución, pero resulta más compleja que la técnica anterior. Destacar en este sentido, el trabajo de [1], donde se compara la agregación de dos riesgos utilizando la metodología de Solvencia II con la teoría de cópulas, en particular, con la cópula de Frank.

- Agregación mediante técnicas de simulación. Permite agregar variables aleatorias sin necesidad de hacer hipótesis iniciales sobre la dependencia entre ellas.

El primer objetivo de este trabajo es proponer un modelo interno, basado en técnicas de simulación, que permita el cálculo del $S C R$ de una compañía para su cartera del ramo de vida, integrada por pólizas que cubren la supervivencia o longevidad del asegurado (rentas de supervivencia), y por pólizas que cubren el fallecimiento de este (seguros). En particular, se utilizará el método de Monte Carlo para simular la cartera de vida de la compañía de seguros, el cual permitirá obtener directamente la agregación de los dos riesgos considerados, sin necesidad de utilizar las matrices de correlación.

Una vez elaborado el modelo interno para la agregación de los riesgos de mortalidad y longevidad del módulo de riesgo de suscripción del seguro de vida, el segundo objetivo es analizar el efecto que tienen las diferentes modalidades de reaseguro, concretamente el reaseguro cuota parte, el reaseguro de excedentes y el reaseguro stop-loss, en el capital de solvencia obligatorio de la compañía de seguros. En la literatura actuarial, existen trabajos que analizan el efecto mitigador del reaseguro en el SCR de la compañía, en este sentido cabe destacar entre otros los trabajos de [2], [3] y [4]. En estos trabajos no se considera el tamaño de la cartera como variable a tener en cuenta en el cálculo del SCR de la compañía y, por tanto, no se tiene en cuenta el riesgo no sistemático de la misma, es decir, el derivado de las fluctuaciones aleatorias de las tasas de mortalidad respecto al valor esperado debido al tamaño de la cartera de la compañía.

El trabajo tiene la siguiente estructura; en el apartado 2 se expone cómo se lleva a cabo la agregación de riesgos en la fórmula estándar según la Directiva de Solvencia II; en el apartado 3 se describe el modelo interno propuesto para la agregación de riesgos; en el apartado 4 se incorpora el reaseguro en el modelo interno propuesto, concretamente se analiza la incorporación del reaseguro cuota parte, del reaseguro de excedentes y del reaseguro stop-loss; en el apartado 5 se lleva a cabo una aplicación numérica y en el apartado 6 se exponen las consideraciones finales.

\section{Agregación de riesgos en Solvencia II}

La fórmula estándar que propone la Directiva de Solvencia II para obtener el SCR tiene un enfoque modular, estableciéndose necesidades de capital para las diferentes categorías de riesgo y agregándose estas mediante matrices de correlación aportadas por el regulador.

La estructura de la fórmula estándar para calcular el $S C R$ total de la compañía se define en el artículo 103 de Directiva de Solvencia II, y responde a la siguiente expresión: 
donde:

- BSCR (Basic Solvency Capital Requirement) es el capital de solvencia obligatorio básico, regulado en el artículo 104 de la Directiva de Solvencia II.

- $S C R$ por riesgo operacional es el capital de solvencia básico por riesgo operacional, regulado en el artículo 107 de la Directiva de Solvencia II.

- Ajuste por provisiones e impuestos diferidos es el importe del ajuste destinado a tener en cuenta la capacidad de absorción de pérdidas de las provisiones técnicas y los impuestos diferidos, regulado en el artículo 108 de la Directiva de Solvencia II.

El artículo 87 del Reglamento Delegado (UE) 2015/35 de la Comisión de 10 de octubre de 2014, por el que se completa la Directiva 2009/138/CE del Parlamento Europeo y del Consejo sobre el acceso a la actividad de seguro y de reaseguro y su ejercicio (Solvencia II), que complementa al artículo 104 de la Directiva de Solvencia II, establece la siguiente fórmula para el cálculo del BSCR:

$$
B S C R=\sqrt{\sum_{i, j} \operatorname{Corr}_{i, j} \cdot S C R_{i} \cdot S C R_{j}}+S C R_{\text {intangibles }}
$$

donde:

- $S C R_{\text {intangibles }}$ representa el capital de solvencia obligatorio frente al riesgo de intangibles. Está regulado en el artículo 203 del Reglamento Delegado (UE) 2015/35.

- $\sqrt{\sum_{i, j} \operatorname{Corr}_{i, j} \cdot S C R_{i} \cdot S C R_{j}}$ es la suma correlacionada de todas las posibles combinaciones « $i, j »$, donde $S C R_{i}$ representa el módulo de riesgo $i$ y $S C R_{j}$ representa el módulo de riesgo $j$. En el cálculo, $S C R_{i}$ y $S C R_{j}$ se sustituyen por:

- $S C R_{\text {distinto de vida }}$, que representa el módulo de riesgo de suscripción del seguro distinto del seguro de vida.

- $S C R_{\text {vida }}$, que representa el módulo de riesgo de suscripción del seguro de vida.

- $S C R_{\text {enfermedad }}$, que representa el módulo riesgo de suscripción del seguro de enfermedad.

- $S C R_{\text {mercado }}$, que representa el módulo riesgo de mercado.

- $S C R_{\text {incumplimiento }}$, que representa el módulo de riesgo de incumplimiento de la contraparte.

Como puede observarse en la expresión (2), el cálculo del BSCR se obtiene por suma correlacionada de todos los capitales de solvencia obligatorios menos para el caso del $S C R_{\text {intangibles }}$, cuya agregación se obtiene por suma aritmética

A su vez, el $S C R$ de cada uno de los cinco módulos de riesgo anteriores, se calcula también como la suma correlacionada de los $S C R$ de los submódulos en los que estos se dividen. En la Figura 1, obtenida del Quantitative Impact Studies 5, QIS5, queda recogida la estructura modular de riesgos para el cálculo del SCR.

En el caso del módulo de riesgo de suscripción del seguro de vida, regulado en el artículo 105 , apartado 3, de la Directiva de Solvencia II, la fórmula estándar del cálculo del $S C R$, establecida en el punto 3 del anexo IV de la Directiva, es la siguiente:

$$
S C R_{v i d a}=\sqrt{\sum_{i, j} \operatorname{Corr}_{i, j} \cdot S C R_{i} \cdot S C R_{j}}
$$

en la que $S C R_{i}$ representa el submódulo $i$ y $S C R_{j}$ representa el submódulo $j$, e « $i, j$, significa que la suma de los diferentes términos debe cubrir todas las combinaciones posibles de $i$ y $j$. En el cálculo, $S C R_{i}$ y $S C R_{j}$ se sustituyen por: 
- $S C R_{\text {mortalidad }}$, que representa el submódulo de riesgo de mortalidad.

- $S C R_{\text {longevidad }}$, que representa el submódulo de riesgo de longevidad.

- $S C R_{\text {discapadidad }}$, que representa el submódulo de riesgo de discapacidad.

- $S C R_{\text {gastos vida }}$, que representa el submódulo de riesgo de gastos del seguro de vida.

- $S C R_{\text {revisión }}$, que representa el submódulo de riesgo de revisión.

- $S C R_{\text {caducidad }}$, que representa el submódulo de riesgo de caducidad.

- $S C R_{\text {catástrofe vida }}$, que representa el submódulo de riesgo de catástrofe del seguro de vida.

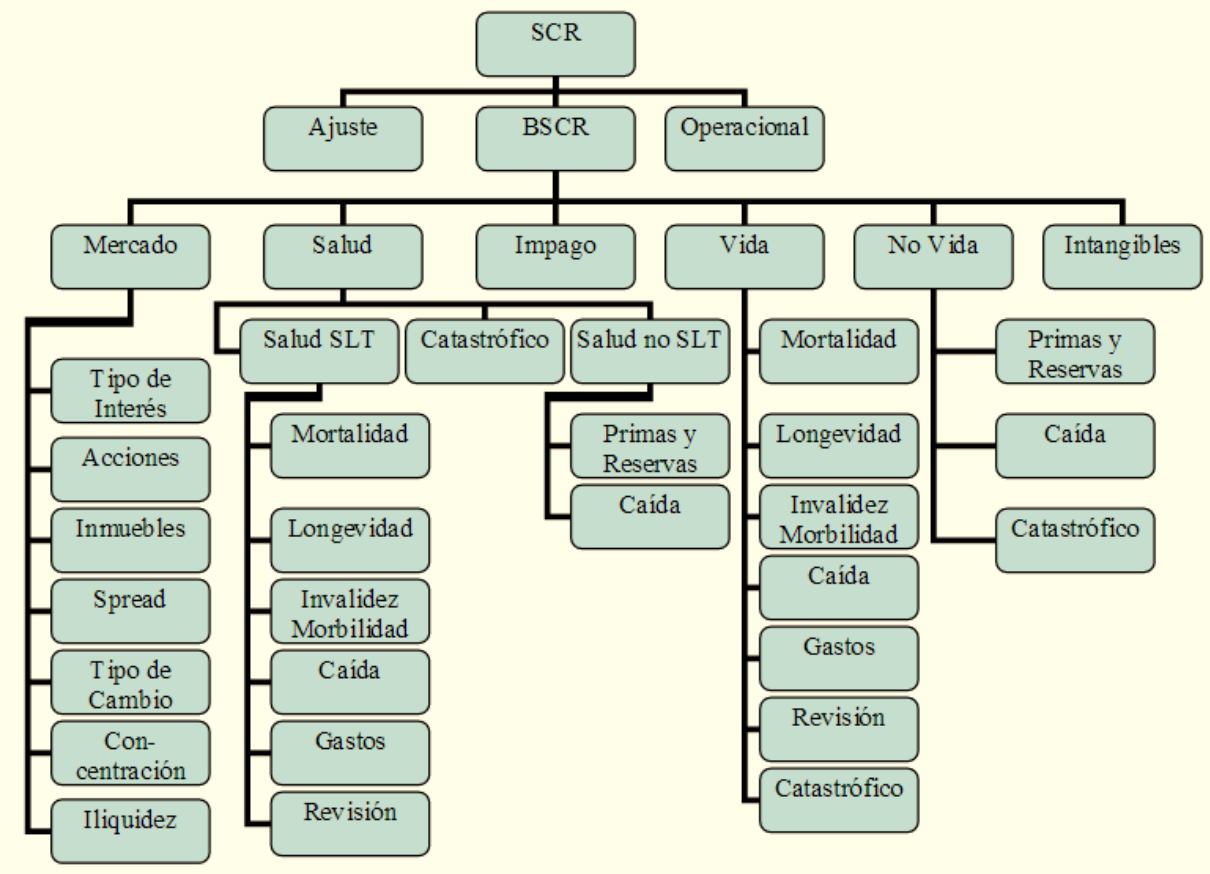

Figura 1. Estructura modular de riesgos para el cálculo del $S C R$

La fórmula estándar del $S C R$ del módulo de riesgo de suscripción del seguro de vida en el caso particular de considerar únicamente los submódulos de riesgo de mortalidad y de riesgo de longevidad es la siguiente:

$$
S C R_{\text {vida }}=\sqrt{S C R_{\text {mort }}^{2}+S C R_{\text {long }}^{2}-0,50 \cdot S C R_{\text {mort }} \cdot S C R_{\text {long }}}
$$

ya que, la matriz de correlaciones, definida en el Reglamento Delegado (UE) 2015/35 de la Comisión de 10 de octubre de 2014 en el artículo 136, punto 3, es para estos dos submódulos de riesgo:

Tabla 1. Matriz de correlaciones

\begin{tabular}{c|c|c}
\hline $\mathrm{j}$ & Mortalidad & Longevidad \\
\hline Mortalidad & 1 & $-0,25$ \\
\hline Longevidad & $-0,25$ & 1 \\
\hline
\end{tabular}

Para obtener el $S C R$ total de cada riesgo, $S C R_{\text {long }}$ y $S C R_{\text {mort }}$, hay que calcular el $S C R$ para cada individuo asociado a cada riesgo.

El $S C R$ para el asegurado $i$, asociado al riesgo de mortalidad, $S C R_{m o r t, i}$, se calcula del siguiente modo en el modelo estándar: 


$$
S C R_{\text {mort }, i}=N A V_{0, \text { mort }, i}-\left(N A V_{0, \text { mort }, i} \mid \text { shock de mortalidad }\right) \text { con } i=1, \ldots, n_{0, \text { seg }}
$$

siendo $n_{0, \text { seg }}$ el número de asegurados del colectivo que tienen una operación de seguro en el momento del análisis, $t=0$, y $N A V_{0, \text { mort }, i}$ la diferencia entre el valor actual de mercado de los activos y los pasivos asociados a la operación de seguro del asegurado $i$.

El shock de mortalidad a aplicar es un incremento del $15 \%$ de la tasa de mortalidad, para cada edad y cada póliza (Artículo 137 del Reglamento Delegado (UE) 2015/35).

Para el riesgo de longevidad, el $S C R$ para el asegurado $i, S C R_{\text {long, } i}$, se obtiene como:

$$
S C R_{\text {long }, i}=N A V_{0, \text { long }, i}-\left(N A V_{0, \text { long }, i} \mid \text { shock de longevidad }\right) \text { con } i=1, \ldots, n_{0, \text { ren }}
$$

siendo $n_{0, \text { ren }}$ el número de asegurados del colectivo que tienen una operación de renta de supervivencia en el momento del análisis, $t=0$, y $N A V_{0, l o n g, i}$ la diferencia entre el valor actual de mercado de los activos y los pasivos asociados a la operación de la renta de supervivencia del asegurado $i$.

En este caso, el shock de longevidad a aplicar es un decremento del $20 \%$ de la tasa de mortalidad, para cada edad y cada póliza (Artículo 138 del Reglamento Delegado (UE) 2015/35).

El SCR total de cada riesgo se obtiene sumando los SCR individuales:

$$
\begin{aligned}
S C R_{\text {mort }} & =\sum_{i=1}^{n_{0, \text { seg }}} S C R_{\text {mort }, i} \\
S C R_{\text {long }} & =\sum_{i=1}^{n_{0, \text { ren }}} S C R_{\text {long }, i}
\end{aligned}
$$

En el caso particular de un colectivo en el que cada asegurado tenga contratado un seguro de vida y una renta de supervivencia, entonces $n_{0}=n_{0, \text { ren }}=n_{0, \text { seg }}$, siendo $n_{0}$ el número de asegurados que integran el colectivo en el momento del análisis, $t=0$.

\section{Modelo interno para la agregación de los riesgos de mortalidad y de longevidad para el módulo del riesgo de suscripción del seguro de vida}

En este apartado se generaliza para los riesgos de mortalidad y longevidad el modelo interno para calcular el SCR propuesto por [5], para el riesgo de mortalidad. Este modelo se basa en simular, por el método de Monte Carlo, la evolución de siniestralidad de la cartera y asume la interpretación formal del artículo 101 de la Directiva de Solvencia II y la formalización matemática propuesta por [6]:

$$
S C R=\operatorname{VaR}_{0,995}\left(N A V_{0}-N A V_{1}\right)=\operatorname{VaR}_{0,995}\left(D N A V_{0}\right)
$$

siendo:

- $N A V_{t}$, con $t=\{0,1\}$, es la variable aleatoria valor actual neto en $t$. Se calcula como la diferencia en $t$ entre el valor de mercado de los activos, $A_{t}$, y de los pasivos, $L_{t}$, esto es, $N A V_{t}=A_{t}-L_{t}$.

- $D N A V_{0}=N A V_{0}-N A V_{1}$.

- $\operatorname{VaR}_{0,995}(X)$ es el valor en riesgo (Value at Risk) de la variable aleatoria $X$ con un nivel de confianza del $99,5 \%$.

En este caso se asume también la hipótesis de que la cartera de la compañía de seguros en el momento del análisis, $t=0$, está constituida por un colectivo $N_{0}$ formado por $n_{0}$ asegurados, pero cada asegurado 
$i$, de edad actuarial $x_{i}$, con $i=1,2, \ldots, n_{0}$, tiene contratado un seguro de vida y/o una renta de supervivencia, cuyas prestaciones y contraprestaciones son conocidas en el momento del análisis, siendo:

$$
\begin{gathered}
N A V_{0}=\sum_{t=0}^{Q}\left(a_{t}-b_{t}\right) \cdot\left[1+I_{1}(0, t)\right]^{-t} \\
N A V_{1}=\sum_{t=1}^{Q}\left(a_{t}-b_{t}\right) \cdot\left[1+I_{1}(1, t)\right]^{-(t-1)}
\end{gathered}
$$

donde:

- $t=0,1,2, \ldots, Q$, es el horizonte temporal de la operación, expresado en años, y $Q$ es la variable aleatoria primer año en el que la compañía de seguros queda liberada del pago de prestaciones a los beneficiarios.

- $a_{t}$ y $b_{t}$, con $t=0,1,2, \ldots, Q$, son, respectivamente, las variables aleatorias cuantía de los activos aportados en $t$ por los asegurados y cuantía de los pasivos satisfechos en $t$. En este modelo los activos vienen dados por las primas y los pasivos por las sumas aseguradas de la operación.

- $I_{1}(0, t)$ y $I_{1}(1, t)$, con $t=0,1,2, \ldots, Q$, son, respectivamente, los tantos de interés efectivos anuales al contado e implícitos, siendo $I_{1}(0,0)=0$. ([7]). Los tantos de interés al contado vienen dados por el regulador.

Para calcular las variables aleatorias $N A V_{0}$ y $N A V_{1}$, es necesario conocer la evolución del colectivo $N_{0}$ en el tiempo. Su evolución se obtiene a partir de la variable aleatoria $T_{N_{0}}$, que proporciona el número de años enteros que van a permanecer con vida cada uno de $\operatorname{los} n_{0}$ asegurados que forman el colectivo $N_{0}$ en el momento $t=0$. Debido al elevado número de realizaciones que tiene la variable aleatoria $T_{N_{0}}$ es imposible trabajar directamente con su función de distribución. Este problema se resuelve simulando, por el método de Monte Carlo, las realizaciones de dicha variable.

Una vez conocida la función de distribución de la variable aleatoria $T_{N_{0}}$, bajo la hipótesis que se llevan a cabo $z$ simulaciones, se obtienen el resto de las variables aleatorias relevantes del modelo, $Q, a_{t}$, $b_{t}$ y $D N A V_{0}$, cuyas realizaciones son, respectivamente:

$$
\begin{gathered}
\left\{Q^{1}, Q^{2}, \ldots, Q^{l}, \ldots, Q^{z}\right\} \\
\left\{a_{t}^{1}, a_{t}^{2}, \ldots, a_{t}^{l}, \ldots, a_{t}^{z}\right\} \\
\left\{b_{t}^{1}, b_{t}^{2}, \ldots, b_{t}^{l}, \ldots, b_{t}^{z}\right\} \\
\left\{D N A V_{0}^{1}, D N A V_{0}^{2}, \ldots, D N A V_{0}^{l}, \ldots, D N A V_{0}^{z}\right\}
\end{gathered}
$$

con $t=0,1,2, \ldots, Q^{l}$ y $l=1,2, \ldots, z$, donde $Q^{l}, a_{t}^{l}, b_{t}^{l}$ y $D N A V_{0}^{l}$ son, respectivamente, las realizaciones de las variables aleatorias $Q, a_{t}, b_{t}$ y $D N A V_{0}$ asociadas a la trayectoria de evolución del colectivo $l$.

Para el módulo del riesgo de suscripción del seguro de vida considerando la agregación del submódulo del riesgo de mortalidad y del submódulo del riesgo de longevidad, las realizaciones $a_{t}^{l}$ y $b_{t}^{l}$ de las variables aleatorias $a_{t}$ y $b_{t}$, asociadas a la trayectoria de evolución del colectivo $l$, con $l=$ $1,2, \ldots, z$, se obtienen respectivamente a partir de:

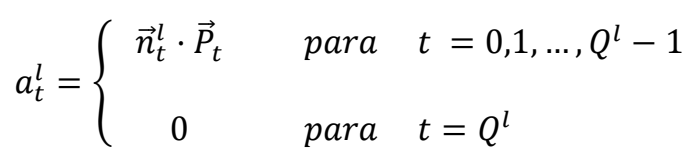




$$
b_{t}^{l}=\left\{\begin{array}{ccl}
b_{0} & \text { para } t=0 \\
\vec{d}_{t}^{l} \cdot \overrightarrow{S_{t}^{s e g}}+\vec{n}_{t}^{l} \cdot \overrightarrow{S_{t}^{r e n}} & \text { para } t=1,2, \ldots, Q^{l}
\end{array}\right.
$$

donde:

- $\vec{P}_{t}$, con $t=0,1,2, \ldots, Q^{l}-1$, es el vector de primas que deben satisfacer en $t$ los asegurados del colectivo inicial $N_{0}$, suponiendo que todos están vivos en $t$.

- $\vec{n}_{t}^{l}$, con $t=0,1,2, \ldots, Q^{l}-1$, es el vector formado por ceros y unos, que tiene tantas componentes como asegurados $n_{0}$ tiene el colectivo $N_{0}$, y muestra qué asegurados están vivos en $t$, dada la trayectoria de evolución del colectivo $l$. Si la componente $i$, con $i=1, \ldots, n_{0}$, vale 1 , indica que el asegurado $i$-ésimo está vivo en $t$, y si vale 0 es que está muerto.

- $b_{0}$ es un valor cierto y vale 0 si el colectivo $N_{0}$ es de nueva creación.

- $\vec{d}_{t}^{l}$, con $t=1, \ldots, Q^{l}$, es un vector formado por ceros y unos, que tiene tantas componentes como asegurados $n_{0}$ tienen el colectivo $N_{0}$, y muestra qué asegurados del colectivo inicial fallecen durante el año $t$, dada la trayectoria de evolución del colectivo $l$. Si la componente $i$, con $i=1, \ldots, n_{0}$, vale 1 , indica que el asegurado $i$-ésimo ha fallecido en el año $t$, y si vale 0 es que permanece vivo en $t$ o ha fallecido en un año diferente de $t$.

- $\overrightarrow{S_{t}^{\text {seg }}}$, con $t=1, \ldots, Q^{l}$, es el vector de la prestación en caso de fallecimiento que tiene que pagar la compañía en $t$ a los beneficiarios de los asegurados del colectivo inicial $N_{0}$, suponiendo que todos los asegurados que tienen contratado un seguro falleciesen en el año $t$.

- $\overrightarrow{S_{t}^{r e n}}$, con $t=1, \ldots, Q^{l}$, es el vector de la prestación en caso de supervivencia que tiene que pagar la compañía en $t$ a los asegurados del colectivo inicial $N_{0}$, suponiendo que todos los asegurados que tienen contratada la renta estén vivos en el año $t$.

Los vectores $\vec{n}_{t}^{l}$ y $\vec{d}_{t}^{l}$ se obtienen a partir de la realización $l$-ésima de la variable aleatoria $T_{N_{0}}$.

La agregación de los dos riesgos considerados en la cartera de la compañía se realiza para cada simulación, directamente en la realización $l$-ésima, $b_{t}^{l}$, de la variable aleatoria pasivos en $t, b_{t}$. De esta manera, $b_{t}^{l}$, tiene en cuenta el total que en $t$, debe pagar la compañía en concepto de rentas y seguros, de acuerdo con la evolución que tenga el colectivo según su $l$-ésima simulación.

Conocidas las realizaciones, por simulación, se podrá estimar la función de distribución de probabilidad de la variable aleatoria $D N A V_{0}$, y el $S C R$ del colectivo se obtendrá como el $\operatorname{VaR}_{0,995}$ de la variable aleatoria $D N A V_{0}$ :

$$
S C R=\operatorname{VaR}_{0,995}\left(D N A V_{0}\right)
$$

\section{El reaseguro en el modelo interno}

En este apartado se generaliza para el riesgo de mortalidad y de longevidad la incorporación en el modelo interno del reaseguro proporcional y no proporcional, propuesto por [8], para el riesgo de mortalidad. Concretamente, cómo se ve afectado el modelo interno para calcular el $S C R$ con la agregación de los riesgos de mortalidad y de longevidad si se incorpora un reaseguro cuota parte, un reaseguro de excedentes y un reaseguro stop-loss ([9]).

En las expresiones que a continuación se desarrollan se asumen dos hipótesis, la primera que el contrato de reaseguro tiene la misma duración que el contrato de la operación pactada con el asegurado, y la segunda hipótesis, que cada asegurado tiene contratada una única póliza, que cubre las dos contingencias, longevidad y mortalidad.

Si se realizan $z$ simulaciones del colectivo, el $S C R$, desde el punto de vista de la cedente, $S C R^{c}=$ $\operatorname{VaR}_{0,995}\left(D N A V_{0}^{c}\right)$, y desde el punto de vista del reasegurador, $S C R^{r}=\operatorname{VaR}_{0,995}\left(D N A V_{0}^{r}\right)$, se obtiene, respectivamente, a partir de las variables aleatorias cuantía de los activos, $a_{t}^{c}$, y de los pasivos, $b_{t}^{c}$, a cargo 
de la cedente en el momento $t$, y a partir de las variables aleatorias cuantía de los activos, $a_{t}^{r}$, y de los pasivos, $b_{t}^{r}$, a cargo del reasegurador en el momento $t$, cuyas realizaciones son:

$$
\begin{aligned}
& a_{t}^{c}=\left\{a_{t}^{c, 1}, a_{t}^{c, 2}, \ldots, a_{t}^{c, l}, \ldots, a_{t}^{c, z}\right\} \text { у } b_{t}^{c}=\left\{b_{t}^{c, 1}, b_{t}^{c, 2}, \ldots, b_{t}^{c, l}, \ldots, b_{t}^{c, z}\right\}, \text { con } t=0,1,2, \ldots, Q^{l} \\
& a_{t}^{r}=\left\{a_{t}^{r, 1}, a_{t}^{r, 2}, \ldots, a_{t}^{r, l}, \ldots, a_{t}^{r, z}\right\} \text { у } b_{t}^{r}=\left\{b_{t}^{r, 1}, b_{t}^{r, 2}, \ldots, b_{t}^{r, l}, \ldots, b_{t}^{r, z}\right\}, \text { con } t=0,1,2, \ldots, Q^{l}
\end{aligned}
$$

siendo $a_{t}=a_{t}^{c}+a_{t}^{r}$ y $b_{t}=b_{t}^{c}+b_{t}^{r}$.

El cálculo de los pasivos, $b_{t}^{c}$ y $b_{t}^{r}$, será inmediato a partir de las características de cada modalidad de reaseguro, sin embargo, la obtención de los activos, $a_{t}^{c}$ y $a_{t}^{r}$, dependerá del criterio de reparto que se establezca entre los asegurados como se verá a continuación.

\subsection{Cálculo de los pasivos en el reaseguro cuota parte}

El reaseguro cuota parte consiste en que la cedente asume una cuota de retención preestablecida, $k$, con $0 \leq k \leq 1$, expresada en tanto por uno, de todas y cada una de las pólizas independientemente de su suma asegurada. En este caso se va a discriminar la cuota de retención de la cedente según el tipo de prestación, seguro o renta, siendo $k^{\text {seg }}$ y $k^{r e n}$, respectivamente, la cuota de retención de la operación de seguro y de la operación de renta, siendo ambas $0 \leq k^{\text {seg }} \leq 1$ y $0 \leq k^{\text {ren }} \leq 1$. Las cuotas de retención permiten determinar la responsabilidad de la cedente y del reasegurador en el importe del siniestro.

En esta modalidad de reaseguro las realizaciones de la variable aleatoria $b_{t}$, para la cedente, $b_{t}^{c, l}$, asociadas a la trayectoria de evolución del colectivo $l, \operatorname{con} l=1,2, \ldots, z$, son:

$$
b_{t}^{c, l}=\left\{\begin{array}{r}
b_{0}^{c} \quad \text { para } t=0 \\
\vec{d}_{t}^{l} \cdot \overrightarrow{S_{t}^{c, s e g}}+\vec{n}_{t}^{l} \cdot \overrightarrow{S_{t}^{c, r e n}} \text { para } t=1,2, \ldots, Q^{l}
\end{array}\right.
$$

donde:

- $b_{0}^{c}$ es un valor cierto y vale 0 si el colectivo $N_{0}$ es de nueva creación.

- $\overrightarrow{S_{t}^{c, s e g}}=\left(S_{1, t}^{c, s e g}, S_{2, t}^{c, \text { seg }}, \ldots, S_{i, t}^{c, s e g}, \ldots, S_{n_{0}, t}^{c, s e g}\right)=k^{\text {seg }} \cdot \overrightarrow{S_{t}^{\text {seg }}}=k^{\text {seg }} \cdot\left(S_{1, t}^{\text {seg }}, S_{2, t}^{\text {seg }}, \ldots, S_{i, t}^{\text {seg }}, \ldots, S_{n_{0}, t}^{\text {seg }}\right)$, con $t=1, \ldots, Q^{l}$, es el vector de sumas aseguradas a cargo de la cedente que tiene que pagar en $t$ a los beneficiarios del colectivo inicial $N_{0}$, suponiendo que todos los asegurados que tienen contratado el seguro falleciesen en el año $t$.

- $\overrightarrow{S_{t}^{c, r e n}}=\left(S_{1, t}^{c, r e n}, S_{2, t}^{c, r e n}, \ldots, S_{i, t}^{c, r e n}, \ldots, S_{n_{0}, t}^{c, r e n}\right)=k^{r e n} \cdot \overrightarrow{S_{t}^{r e n}}=k^{r e n} \cdot\left(S_{1, t}^{r e n}, S_{2, t}^{r e n}, \ldots, S_{i, t}^{r e n}, \ldots, S_{n_{0}, t}^{r e n}\right)$, con $t=1, \ldots, Q^{l}$, es el vector de sumas aseguradas a cargo de la cedente que tiene que pagar en $t$ a los asegurados del colectivo inicial $N_{0}$, suponiendo que todos los asegurados que tienen contratada la renta estén vivos en el año $t$.

Por tanto,

$$
b_{t}^{c, l}=\left\{\begin{array}{r}
b_{0}^{c} \quad \text { para } t=0 \\
\vec{d}_{t}^{l} \cdot k^{\text {seg }} \cdot \overrightarrow{S_{t}^{\text {seg }}}+\vec{n}_{t}^{l} \cdot k^{r e n} \cdot \overrightarrow{S_{t}^{r e n}} \text { para } t=1,2, \ldots, Q^{l}
\end{array}\right.
$$

Análogamente, desde el punto de vista del reasegurador, las realizaciones $b_{t}^{r, l}$ de la variable aleatoria, $b_{t}^{r}$, asociadas a la trayectoria de evolución del colectivo $l$, con $l=1,2, \ldots, z$, se obtienen a partir de la siguiente expresión: 


$$
b_{t}^{r, l}=\left\{\begin{array}{c}
b_{0}^{r} \quad \text { para } t=0 \\
\vec{d}_{t}^{l} \cdot \overrightarrow{S_{t}^{r, s e g}}+\vec{n}_{t}^{l} \cdot \overrightarrow{S_{t}^{r, r e n}} \text { para } t=1,2, \ldots, Q^{l}
\end{array}\right.
$$

donde:

- $b_{0}^{r}$ es un valor cierto y vale 0 si el colectivo $N_{0}$ es de nueva creación.

- $\overrightarrow{S_{t}^{r, s e g}}=\left(S_{1, t}^{r, s e g}, S_{2, t}^{r, s e g}, \ldots, S_{i, t}^{r, \text { seg }}, \ldots, S_{n_{0}, t}^{r, \text { seg }}\right)=\left(1-k^{\text {seg }}\right) \cdot \overrightarrow{S_{t}^{\text {seg }}}=\left(1-k^{\text {seg }}\right)$. $\left(S_{1, t}^{\text {seg }}, S_{2, t}^{\text {seg }}, \ldots, S_{i, t}^{\text {seg }}, \ldots, S_{n_{0}, t}^{\text {seg }}\right)$, con $t=1, \ldots, Q^{l}$, es el vector de sumas aseguradas a cargo del reasegurador que tiene que pagar en $t$ a los beneficiarios del colectivo inicial $N_{0}$, suponiendo que todos los asegurados que tienen contratado el seguro falleciesen en el año $t$.

- $\overrightarrow{S_{t}^{r, r e n}}=\left(S_{1, t}^{r, r e n}, S_{2, t}^{r, r e n}, \ldots, S_{i, t}^{r, r e n}, \ldots, S_{n_{0}, t}^{r, \text { ren }}\right)=\left(1-k^{r e n}\right) \cdot \overrightarrow{S_{t}^{r e n}}=\left(1-k^{r e n}\right)$. $\left(S_{1, t}^{r e n}, S_{2, t}^{r e n}, \ldots, S_{i, t}^{r e n}, \ldots, S_{n_{0}, t}^{r e n}\right)$, con $t=1, \ldots, Q^{l}$, es el vector de sumas aseguradas a cargo del reasegurador que tiene que pagar en $t$ a los asegurados del colectivo inicial $N_{0}$, suponiendo que todos los asegurados que tienen contratada la renta estén vivos en el año $t$.

De manera que:

$$
b_{t}^{r, l}=\left\{\begin{array}{rr}
b_{0}^{r} \quad \text { para } t=0 \\
\vec{d}_{t}^{l} \cdot\left(1-k^{s e g}\right) \cdot \overrightarrow{S_{t}^{s e g}}+\vec{n}_{t}^{l} \cdot\left(1-k^{r e n}\right) \cdot \overrightarrow{S_{t}^{r e n}} & \text { para } t=1,2, \ldots, Q^{l}
\end{array}\right.
$$

En el caso particular que $k^{r e n}=k^{\text {seg }}=k$ se mantiene la proporcionalidad en las primas y los activos se obtienen de forma inmediata ([8]).

\subsection{Cálculo de los pasivos en el reaseguro de excedentes}

En el reaseguro de excedente, a diferencia del reaseguro cuota parte, la cuota de retención de la cedente es variable para cada póliza y depende de la relación entre el pleno de retención y su suma asegurada. El pleno de retención se va a diferenciar por el tipo de prestación, según sea un seguro o una renta, siendo $M_{i}^{\text {seg }}$ y $M_{i}^{\text {ren }}$, respectivamente, el pleno de retención de la cedente asociado a un seguro o a una renta, de la póliza $i$, con $i=1, \ldots, n_{0}$.

Las realizaciones de la variable aleatoria $b_{t}$, para la cedente, $b_{t}^{c, l}$, asociadas a la trayectoria de evolución del colectivo $l$, con $l=1,2, \ldots, z$, son:

$$
b_{t}^{c, l}=\left\{\begin{array}{c}
b_{0}^{c} \quad \text { para } t=0 \\
\vec{d}_{t}^{l} \cdot \overrightarrow{S_{t}^{c, s e g}}+\vec{n}_{t}^{l} \cdot \overrightarrow{S_{t}^{c, r e n}} \text { para } t=1,2, \ldots, Q^{l}
\end{array}\right.
$$

donde:

- $\overrightarrow{S_{t}^{c, s e g}}=\left(S_{1, t}^{c, s e g}, S_{2, t}^{c, s e g}, \ldots, S_{i, t}^{c, \text { seg }}, \ldots, S_{n_{0}, t}^{c, \text { seg }}\right)$ y $S_{i, t}^{c, \text { seg }}$, para $i=1, \ldots, n_{0}$, se obtiene de la siguiente forma:

$$
S_{i, t}^{c, s e g}=\left\{\begin{array}{lll}
S_{i, t}^{\text {seg }} & \text { si } & S_{i, t}^{\text {seg }}<M_{i}^{\text {seg }} \\
M_{i}^{\text {seg }} & \text { si } & S_{i, t}^{\text {seg }} \geq M_{i}^{\text {seg }}
\end{array}\right.
$$

- $\overrightarrow{S_{t}^{c, r e n}}=\left(S_{1, t}^{c, \text { ren }}, S_{2, t}^{c, r e n}, \ldots, S_{i, t}^{c, r e n}, \ldots, S_{n_{0}, t}^{c, r e n}\right)$ y $S_{i, t}^{c, r e n}$, para $i=1, \ldots, n_{0}$, se obtiene a partir de: 


$$
S_{i, t}^{c, r e n}=\left\{\begin{array}{lll}
S_{i, t}^{r e n} & \text { si } & S_{i, t}^{r e n}<M_{i}^{r e n} \\
M_{i}^{r e n} & \text { si } & S_{i, t}^{r e n} \geq M_{i}^{r e n}
\end{array}\right.
$$

Desde el punto de vista del reasegurador, las realizaciones $b_{t}^{r, l}$ de la variable aleatoria $b_{t}^{r}$ asociadas a la trayectoria de evolución del colectivo $l$, con $l=1,2, \ldots, z$, se obtienen a partir de la siguiente expresión:

$$
b_{t}^{r, l}=\left\{\begin{array}{rrr}
b_{0}^{r} & \text { para } t & =0 \\
\vec{d}_{t}^{l} \cdot \overrightarrow{S_{t}^{r, s e g}}+\vec{n}_{t}^{l} \cdot \overrightarrow{S_{t}^{r, r e n}} & \text { para } t=1,2, \ldots, Q^{l}
\end{array}\right.
$$

donde:

- $\overrightarrow{S_{t}^{r, s e g}}=\left(S_{1, t}^{r, s e g}, S_{2, t}^{r, s e g}, \ldots, S_{i, t}^{r, s e g}, \ldots, S_{n_{0}, t}^{r, s e g}\right)$ y $S_{i, t}^{r, s e g}$, para $i=1, \ldots, n_{0}$, se obtiene de la siguiente forma:

$$
S_{i, t}^{r, s e g}=\left\{\begin{array}{lll}
0 & \text { si } & S_{i, t}^{\text {seg }}<M_{i}^{\text {seg }} \\
S_{i, t}^{\text {seg }}-M_{i}^{\text {seg }} & \text { si } & S_{i, t}^{\text {seg }} \geq M_{i}^{\text {seg }}
\end{array}\right.
$$

- $\overrightarrow{S_{t}^{r, r e n}}=\left(S_{1, t}^{r, r e n}, S_{2, t}^{r, r e n}, \ldots, S_{i, t}^{r, r e n}, \ldots, S_{n_{0}, t}^{r, r e n}\right)$ y $S_{i, t}^{r, \text { ren }}$, para $i=1, \ldots, n_{0}$, se obtiene a partir de:

$$
S_{i, t}^{r, r e n}=\left\{\begin{array}{lll}
0 & \text { si } & S_{i, t}^{r e n}<M_{i}^{r e n} \\
S_{i, t}^{r e n}-M_{i}^{r e n} & \text { si } & S_{i, t}^{r e n} \geq M_{i}^{r e n}
\end{array}\right.
$$

\subsection{Cálculo de los pasivos en el reaseguro stop-loss}

El reaseguro stop-loss se trata de una modalidad de reaseguro no proporcional que se caracteriza porque el reasegurador se obliga a cubrir total o parcialmente el exceso de siniestralidad ocurrido en un año respecto a una prioridad $M$. En esta modalidad de reaseguro no hay proporcionalidad en la distribución de responsabilidades, debido a que el compromiso de las partes depende de la siniestralidad y no de la suma asegurada de las pólizas.

$\mathrm{Si}$ se asume la hipótesis de que la responsabilidad que asume el reasegurador es ilimitada y la prioridad depende del año $t, M_{t}$, entonces las realizaciones de la variable aleatoria $b_{t}$ para la cedente, $b_{t}^{c, l}$, y para el reasegurador, $b_{t}^{r, l}$, asociadas a la trayectoria de evolución del colectivo $l$, con $l=1,2, \ldots, z$, y para $t=1,2, \ldots, Q^{l}$, son:

$$
\begin{gathered}
b_{t}^{c, l}=\left\{\begin{array}{lll}
\vec{d}_{t}^{l} \cdot \overrightarrow{S_{t}^{\text {seg }}}+\vec{n}_{t}^{l} \cdot \overrightarrow{S_{t}^{r e n}} & \text { si } & \vec{d}_{t}^{l} \cdot \overrightarrow{S_{t}^{\text {seg }}}+\vec{n}_{t}^{l} \cdot \overrightarrow{S_{t}^{r e n}} \leq M_{t} \\
M_{t} & \text { si } & \vec{d}_{t}^{l} \cdot \overrightarrow{S_{t}^{\text {seg }}}+\vec{n}_{t}^{l} \cdot \overrightarrow{S_{t}^{r e n}}>M_{t}
\end{array}\right. \\
b_{t}^{r, l}= \begin{cases}0 & \text { si } \vec{d}_{t}^{l} \cdot \overrightarrow{S_{t}^{\text {seg }}}+\vec{n}_{t}^{l} \cdot \overrightarrow{S_{t}^{r e n}} \leq M_{t} \\
\vec{d}_{t}^{l} \cdot \overrightarrow{S_{t}^{\text {seg }}}+\vec{n}_{t}^{l} \cdot \overrightarrow{S_{t}^{r e n}}-M_{t} & \text { si } \quad \vec{d}_{t}^{l} \cdot \overrightarrow{S_{t}^{\text {seg }}}+\vec{n}_{t}^{l} \cdot \overrightarrow{S_{t}^{r e n}}>M_{t}\end{cases}
\end{gathered}
$$

siendo $b_{t}^{c, l}=b_{0}^{c}$ y $b_{t}^{r, l}=b_{0}^{r}$ para $t=0$. 


\subsection{Cálculo de los activos en las diferentes modalidades de reaseguro}

Para poder calcular las realizaciones $a_{t}^{c, l}$ y $a_{t}^{r, l}$ de las variables aleatorias $a_{t}^{c}$ y $a_{t}^{r}$ asociadas a la trayectoria de evolución del colectivo $l, \operatorname{con} l=1,2, \ldots, z$ :

$$
\begin{aligned}
& a_{t}^{c, l}=\left\{\begin{array}{ccl}
\vec{n}_{t}^{l} \cdot \overrightarrow{P_{t}^{c}} & \text { para } t=0,1, \ldots, Q^{l}-1 \\
0 & \text { para } t=Q^{l}
\end{array}\right. \\
& a_{t}^{r, l}=\left\{\begin{array}{ccl}
\vec{n}_{t}^{l} \cdot \overrightarrow{P_{t}^{r}} & \text { para } t=0,1, \ldots, Q^{l}-1 \\
0 & \text { para } & t=Q^{l}
\end{array}\right.
\end{aligned}
$$

es necesario conocer el vector de primas que retiene la cedente, $\overrightarrow{P_{t}^{c}}$, y el vector de primas del reasegurador $\overrightarrow{P_{t}^{r}}$, siendo $\overrightarrow{P_{t}^{c}}=\left(P_{1, t}^{c}, P_{2, t}^{c}, \ldots, P_{i, t}^{c}, \ldots, P_{n_{0}, t}^{c}\right)$ y $\overrightarrow{P_{t}^{r}}=\left(P_{1, t}^{r}, P_{2, t}^{r}, \ldots, P_{i, t}^{r}, \ldots, P_{n_{0}, t}^{r}\right)$, donde $P_{i, t}^{c}$ es la prima que retiene la cedente satisfecha en el año $t$, correspondiente al asegurado $i$, y $P_{i, t}^{r}$ es la prima de reaseguro satisfecha en el año $t$, correspondiente al asegurado $i$.

Tal y como están diseñadas las modalidades de reaseguro analizadas, el cálculo de $P_{i, t}^{c}$ y $P_{i, t}^{r}$ no es inmediato, ya que no hay proporcionalidad a priori sobre las primas retenidas por la cedente y por el reasegurador. El primer paso es obtener la prima única total para toda la cartera, neta de reaseguro, $\boldsymbol{\pi}, \mathrm{y}$ qué parte de esta retiene la cedente, si se considera el reaseguro, $\boldsymbol{\pi}^{\boldsymbol{c}}$. Estas dos primas únicas totales se obtienen a partir de los pasivos. Posteriormente, aplicando criterios de reparto se determinan las primas que retiene la cedente y el reasegurador correspondientes a cada asegurado del colectivo.

El proceso de cálculo de $P_{i, t}^{c}$, y $P_{i, t}^{r}$, con $i=1, \ldots, n_{0}$ y $t=0,1, \ldots, Q^{l}-1$, se puede ver en [8], donde se asume como criterio de reparto para cada asegurado, la proporcionalidad entre la prima que retiene la cedente, $P_{i, t}^{c}$, y la prima de reaseguro, $P_{i, t}^{r}$, con la prima neta de reaseguro $P_{i, t}$, obteniendo las siguientes expresiones:

$$
P_{i, t}^{c}=\gamma \cdot P_{i, t}=\frac{\boldsymbol{\pi}^{\boldsymbol{c}}}{\boldsymbol{\pi}} \cdot P_{i, t} \quad y \quad P_{i, t}^{r}=(1-\gamma) \cdot P_{i, t}=\left(1-\frac{\boldsymbol{\pi}^{\boldsymbol{c}}}{\boldsymbol{\pi}}\right) \cdot P_{i, t}
$$

siendo:

- $\gamma$ : coeficiente de proporcionalidad.

- $P_{i, t}$ : prima neta de reaseguro satisfecha en el año $t$ correspondiente al asegurado $i$.

- $\pi$ : prima única total de la cartera neta de reaseguro.

- $\boldsymbol{\pi}^{c}$ : prima única total de la cartera que retiene la cedente.

Una vez calculadas las primas $P_{i, t}^{c}$ y $P_{i, t}^{r}$, se obtienen por simulación las realizaciones $a_{t}^{c, l}$ y $a_{t}^{r, l}$ de las variables aleatorias $a_{t}^{c}$ y $a_{t}^{r}$ asociadas a la trayectoria de evolución del colectivo $l$, con $l=1,2, \ldots, z$.

\section{Aplicación numérica}

En este apartado se ilustra un ejemplo numérico del cálculo del $S C R$ del riesgo de suscripción de vida, para una cartera formada por seguros de vida y rentas de supervivencia bajo dos supuestos:

- Supuesto 1: Sin reaseguro. En este caso se obtiene el SCR de la compañía de seguros utilizando el modelo interno propuesto en el apartado 3 y comparándolo con el modelo estándar descrito en el apartado 2.

- Supuesto 2: Con reaseguro. En este caso se calcula el SCR desde el punto de vista de la cedente y del reasegurador para las tres modalidades de reaseguro analizadas, utilizando el modelo interno propuesto en el apartado 4. 
En los dos supuestos se asume que la cartera de la compañía de seguros está formada por un colectivo homogéneo en cuanto a edades, sexo y características de la operación, y que la cartera es de nueva creación. El colectivo está formado por individuos de sexo masculino, con edad actuarial $x=60$ años, donde cada uno de ellos tiene contratada una operación mixta integrada por un seguro inmediato, temporal de 15 años y con una suma asegurada, $S=2.000 €$, y una renta anual, anticipada, diferida 15 años y temporal 15 años, de cuantía constante de $200 €$. La operación mixta está pactada a primas periódicas anuales hasta la temporalidad del seguro, 15 años.

Los datos técnicos asumidos en la operación son los siguientes:

- Tipo de interés técnico del $2 \%$ efectivo anual: $I_{1}=0,02$.

- Tablas de mortalidad: Población asegurada española masculina PASEM 2010.

- Estructura de tipos de interés al contado libres de riesgo 31-05-2018 (EIOPA).

Al ser una cartera de nueva creación, la cuantía de los pasivos satisfechos en $t=0$ por la compañía de seguros es cero, $b_{0}=0$.

El importe de la prima periódica anual, $P$, a pagar por cada asegurado de edad actuarial $x=60$ años, se obtiene a partir de la siguiente expresión:

$$
P=\frac{2.000 \cdot \sum_{t=0}^{14} t / q_{60} \cdot(1+0,02)^{-(t+1)}+200 \cdot \sum_{t=15}^{29} t P_{60} \cdot(1+0,02)^{-t}}{\sum_{t=0}^{14} t P_{60} \cdot(1+0,02)^{-t}}=107,90 €
$$

donde $t / q_{60}$ es la probabilidad de que una persona de edad actuarial $x=60$ años viva $t$ años y fallezca en el año siguiente y $t P_{60}$ es la probabilidad que una persona de edad actuarial 60 años viva $t$ años más.

Para esta cartera los vectores de primas y cuantías aseguradas, $\vec{P}_{t}, \overrightarrow{S_{t}^{s e g}}$ y $\overrightarrow{S_{t}^{r e n t a}}$ son, respectivamente:

$$
\begin{gathered}
\vec{P}_{t}=(107,90 ; 107,90 ; \ldots ; 107,90) \text { con } t=0,1,2, \ldots, 14 \\
\overrightarrow{S_{t}^{\text {seg }}}= \begin{cases}(2.000,2.000, \ldots, 2000) & \text { para } t=1,2,3,4, \ldots, 15 \\
(0,0, \ldots, 0) & \text { para } t=16,17, \ldots, 30\end{cases} \\
\overrightarrow{S_{t}^{r e n}}= \begin{cases}(0,0, \ldots, 0) & \text { para } t=1,2,3, \ldots, 14 \\
(200,200, \ldots, 200) & \text { para } t=15,16, \ldots, 29\end{cases}
\end{gathered}
$$

donde el número de componentes de cada vector coincide con el tamaño del colectivo.

Los cálculos del SCR obtenidos aplicando el método interno propuesto se han realizado en lenguaje de programación $R$ y con 100.000 simulaciones.

Todas las tablas y figuras que aparecen a continuación son de elaboración propia.

\subsection{Supuesto 1: Sin reaseguro}

En la Tabla 2 se muestra, para el modelo interno propuesto y el modelo estándar, y para diferentes tamaños del colectivo $n_{0}$, el capital de solvencia obligatorio total para el ramo de vida, $S C R$, y el que corresponde a cada individuo $i$ perteneciente al colectivo $n_{0}, S C R_{i}$, que en este ejemplo, al tratarse de un colectivo homogéneo se obtiene a partir de:

$$
S C R_{i}=\frac{S C R}{n_{0}} \quad \operatorname{con} i=1, \ldots, n_{0}
$$


Tabla 2. SCR modelo interno versus SCR modelo estándar

\begin{tabular}{c|c|c|c|c|c|c|c|c}
\hline Tamaño colectivo $n_{0}$ & $\mathbf{1}$ & $\mathbf{1 0}$ & $\mathbf{1 0 0}$ & $\mathbf{5 0 0}$ & $\mathbf{1 . 0 0 0}$ & $\mathbf{2 . 0 0 0}$ & $\mathbf{3 . 0 0 0}$ & $\mathbf{5 . 0 0 0}$ \\
\hline Modelo interno & & & & & & & & \\
\hline$S C R$ & 73,80 & 724,87 & $7.179,35$ & 35.672 & $65.342,36$ & $124.681,80$ & $181.948,40$ & 292.816 \\
\hline$S C R_{i}$ & 73,80 & 72,49 & 71,79 & 71,34 & 65,34 & 62,34 & 60,65 & 58,56 \\
\hline Modelo estándar & & & & & & & & \\
\hline$S C R_{\text {long }}$ & 39,58 & 395,75 & $3.957,52$ & 19.79 & $39.575,19$ & $79.150,38$ & $118.725,60$ & 197.876 \\
\hline$S C R_{\text {mort }}$ & 65,39 & 653,90 & 6.539 & 32.695 & 65.390 & 130.780 & 196.170 & 326.950 \\
\hline$S C R$ & 67,44 & 674,40 & $6.743,99$ & 33.720 & $67.439,88$ & $134.879,75$ & $202.319,60$ & $337.199,40$ \\
\hline$S C R_{i}$ & 67,44 & 67,44 & 67,44 & 67,44 & 67,44 & 67,44 & 67,44 & 67,44 \\
\hline
\end{tabular}

Como puede apreciarse en la Tabla 2, en el modelo estándar se contempla un beneficio por diversificación ya que $S C R<S C R_{\text {long }}+S C R_{\text {mort }}$ debido a las correlaciones negativas que Solvencia II asume para los dos riesgos considerados.

El comportamiento del $S C R$ queda representado en la Figura 2, donde se aprecia que el $S C R$ obtenido por el método estándar, proporciona valores menores para colectivos pequeños que el $S C R$ calculado con el modelo interno.

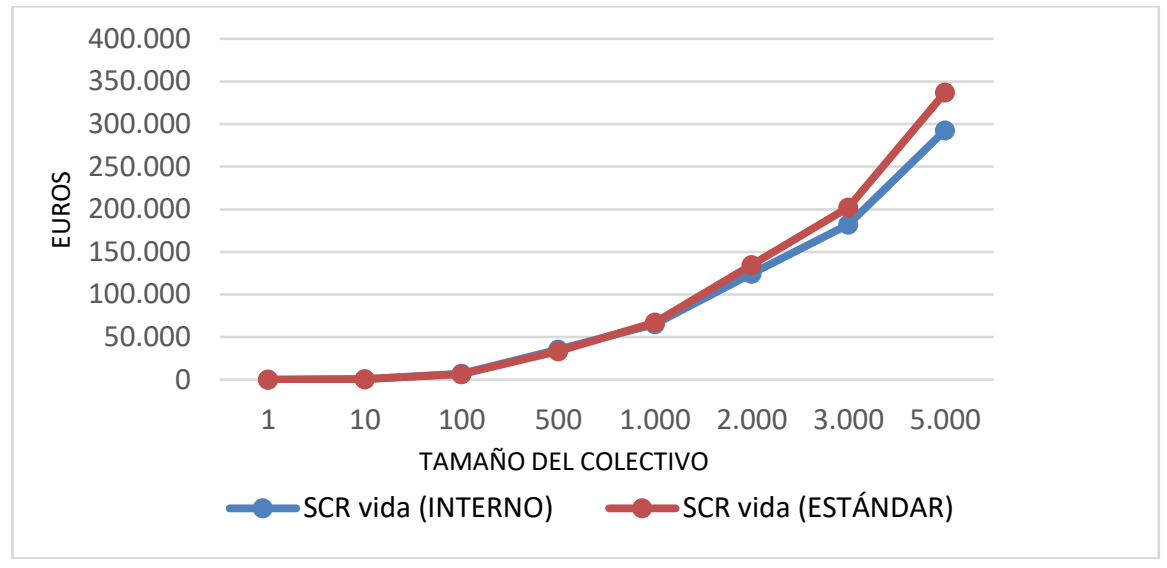

Figura 2. SCR modelo interno y estándar según el tamaño del colectivo

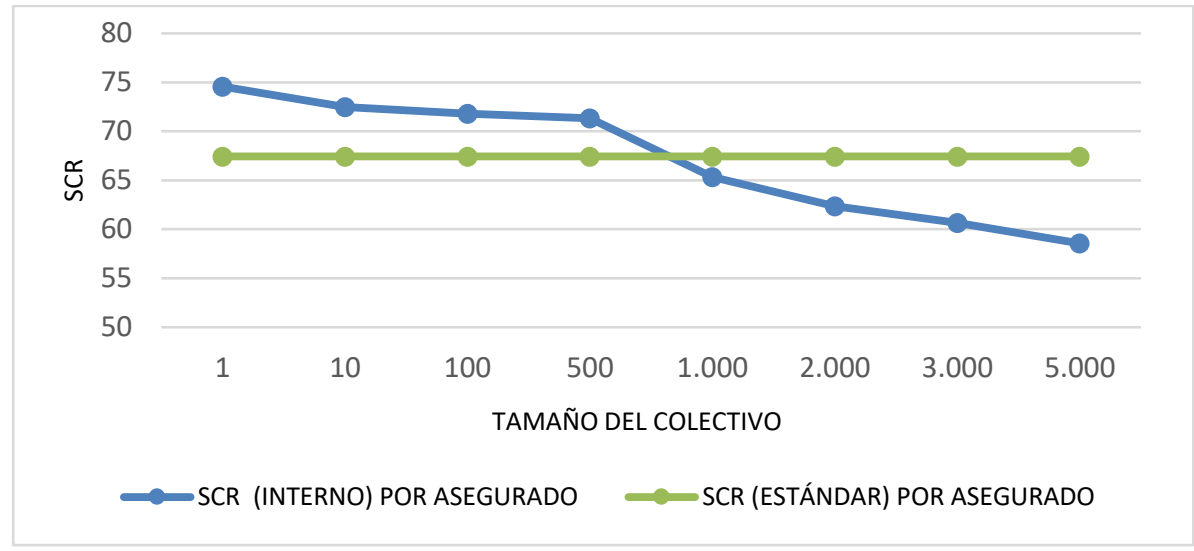

Figura 3. SCR individual según el tamaño del colectivo

En la Figura 3 se representa el $S C R_{i}$ obtenido para cada modelo según el tamaño del colectivo. El modelo estándar proporciona un valor del $S C R_{i}$ que es independiente del tamaño del colectivo, a 
diferencia de lo que sucece con el modelo interno, el cual se va haciendo más pequeño conforme aumenta el tamaño del colectivo. Este comportamiento tiene que ver con la disminución que se produce en el riesgo no sistemático del colectivo cuando este aumenta de tamaño.

\subsection{Supuesto 2: Con reaseguro}

A continuación se obtienen los resultados para el $S C R^{c}, S C R^{r}$ y el $S C R^{\text {Total }}$, calculado este último como suma de los dos primeros, bajo la hipótesis de que la cedente tiene contratado una de las siguientes modalidades de reaseguro: cuota parte, excedente y stop-loss. También se calcula el $S C R_{i}^{c}$, el $S C R_{i}^{r}$ y el $S C R_{i}^{\text {Total }}$ de cada asegurado $i$, que, en este ejemplo, al tratarse de un colectivo homogéneo, se obtienen a partir de:

$$
S C R_{i}^{c}=\frac{S C R^{c}}{n_{0}} ; S C R_{i}^{r}=\frac{S C R^{r}}{n_{0}} ; \quad S C R_{i}^{\text {Total }}=\frac{S C R^{\text {Total }}}{n_{0}} \operatorname{con} i=1, \ldots, n_{0}
$$

\subsubsection{Reaseguro cuota parte}

En la Tabla 3 se muestran los valores de $\gamma, S C R^{c}, S C R^{r}, S C R^{\text {Total }}, S C R_{i}^{c}, S C R_{i}^{r}$ y $S C R_{i}^{\text {Total }}$, para un contrato de reaseguro cuota parte con unas cuotas de retención $k^{\text {seg }}=0,6$ y $k^{r e n}=0,3$, bajo diferentes escenarios de tamaño del colectivo, $n_{0}$.

Tabla 3. Reaseguro cuota parte con $k^{\text {seg }}=0,6$ y $k^{\text {ren }}=0,3$

\begin{tabular}{c|c|c|c|c|c|c|c}
\hline $\boldsymbol{n}_{\mathbf{0}}$ & $\boldsymbol{\gamma}$ & $\boldsymbol{S C R}^{\boldsymbol{c}}$ & $\boldsymbol{S C R}^{\boldsymbol{r}}$ & $\boldsymbol{S C R}^{\text {Total }}$ & $\boldsymbol{S C R}_{\boldsymbol{i}}^{\boldsymbol{c}}$ & $\boldsymbol{S C R}_{\boldsymbol{i}}^{\boldsymbol{r}}$ & $\boldsymbol{S C R}_{\boldsymbol{i}}^{\text {Total }}$ \\
\hline 1 & 0,3963311 & 43,98 & 66,88 & 110,86 & 43,98 & 66,88 & 110,86 \\
\hline 10 & 0,3963311 & 433,76 & 657,17 & $1.090,93$ & 43,38 & 65,72 & 109,09 \\
\hline 50 & 0,3963311 & $2.150,38$ & $3.263,25$ & $5.413,62$ & 43,01 & 65,26 & 108,27 \\
\hline 100 & 0,3963311 & $4.289,28$ & $6.515,40$ & $10.804,67$ & 42,89 & 65,15 & 108,05 \\
\hline 500 & 0,3963311 & $21.318,57$ & $32.462,74$ & $53.781,31$ & 42,64 & 64,93 & 107,56 \\
\hline 1.000 & 0,3963311 & $39.075,86$ & $62.560,99$ & $101.636,85$ & 39,08 & 62,56 & 101,64 \\
\hline 1.500 & 0,3963311 & $56.785,71$ & $92.623,99$ & $149.409,70$ & 37,86 & 61,75 & 99,61 \\
\hline
\end{tabular}

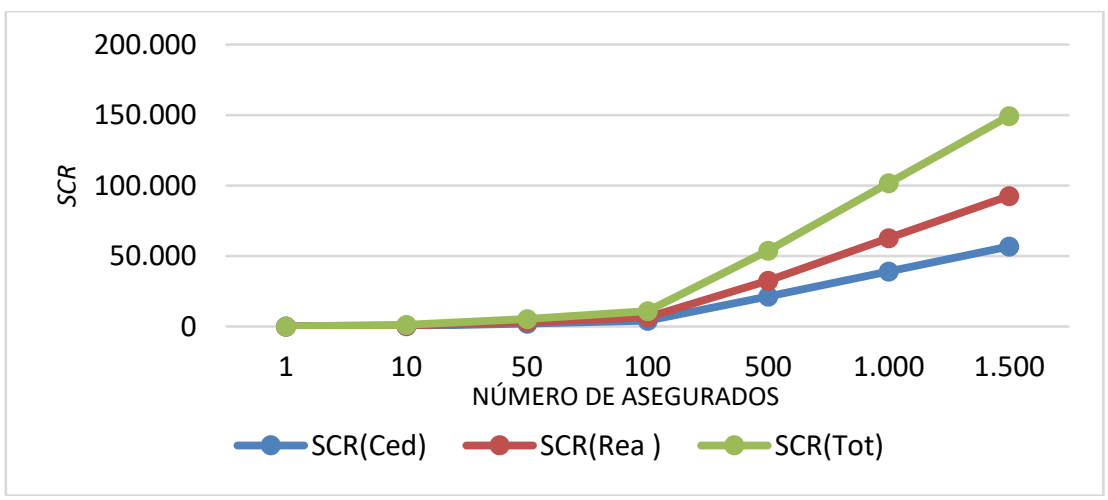

Figura 4: Para $k^{\text {seg }}=0,6$ y $k^{\text {ren }}=0,3$ relación entre $S C R^{c}, S C R^{r}, S C R^{\text {Total }}$ y $n_{0}$

Para $k^{\text {seg }}=0,6$ y $k^{r e n}=0,3$ el valor del parámetro $\gamma=0,3963311$ es independiente del tamaño del colectivo. Como cabe esperar, hay una relación directa entre el $S C R^{c}$ y el $S C R^{r}$ con el tamaño del colectivo, sin embargo, esta relación es inversa cuando se considera el $S C R_{i}^{c}$ y el $S C R_{i}^{r}$, es decir, cuanto mayor es el colectivo, menor es lo que tiene que dotar la cedente y el reasegurador por asegurado, ya que 
disminuye el riesgo no sistemático de la cartera. Estos comportamientos quedan ilustrados en la Figura 4 y en la Figura 5, respectivamente.

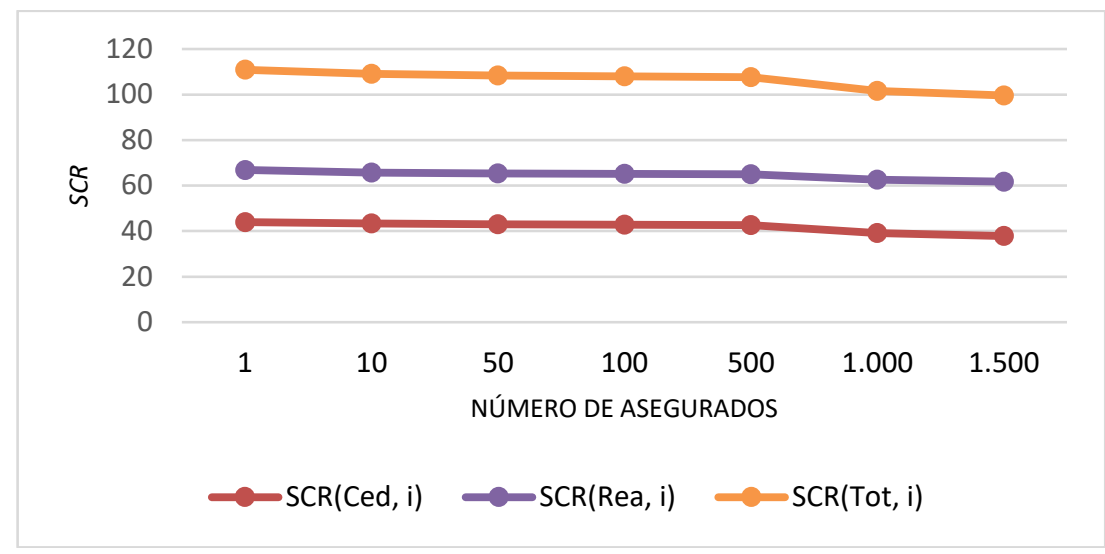

Figura 5: Para $k^{s e g}=0,6$ y $k^{r e n}=0,3$ relación entre $S C R_{i}^{c}, S C R_{i}^{r}, S C R_{i}^{\text {Total }}$ y $n_{0}$

\subsubsection{Reaseguro de excedentes}

En la Tabla 4 se muestra el valor del parámetro $\gamma$, y los valores de $S C R^{c}, S C R^{r}, S C R^{\text {Total }}, S C R_{i}^{c}, S C R_{i}^{r}$ y $S C R_{i}^{\text {Total }}$ para un contrato de reaseguro de excedentes con los siguientes plenos de retención $M^{\text {seg }}=$ $1.000 €$ y $M^{r e n}=150 €$, bajo diferentes escenarios de tamaño del colectivo, $n_{0}$.

Tabla 4. Reaseguro de excedente con $M^{\text {seg }}=1.000 €$ y $M^{\text {ren }}=150 €$

\begin{tabular}{c|c|c|c|c|c|c|c}
\hline $\boldsymbol{n}_{\mathbf{0}}$ & $\boldsymbol{\gamma}$ & $\boldsymbol{S C R}^{\boldsymbol{c}}$ & $\boldsymbol{S C R ^ { \boldsymbol { r } }}$ & $\boldsymbol{S C R} \mathbf{R}^{\text {Total }}$ & $\boldsymbol{S C R}_{\boldsymbol{i}}^{\boldsymbol{c}}$ & $\boldsymbol{S C R}_{\boldsymbol{i}}^{\boldsymbol{r}}$ & $\boldsymbol{S C R}_{\boldsymbol{i}}^{\text {Total }}$ \\
\hline 1 & 0,6698002 & 74,22 & 36,64 & 110,86 & 74,22 & 36,64 & 110,86 \\
\hline 10 & 0,6698002 & 729,11 & 361,29 & $1.090,40$ & 72,91 & 36,13 & 109,04 \\
\hline 50 & 0,6698002 & $3.621,10$ & $1.790,61$ & $5.411,71$ & 72,42 & 35,81 & 108,23 \\
\hline 100 & 0,6698002 & $7.229,53$ & $3.572,30$ & $10.801,83$ & 72,30 & 35,72 & 108,02 \\
\hline 500 & 0,6698002 & $36.015,34$ & $17.762,15$ & $53.777,49$ & 72,03 & 35,52 & 107,55 \\
\hline 1.000 & 0,6698002 & $69.078,94$ & $32.556,56$ & $101.635,50$ & 69,08 & 32,56 & 101,64 \\
\hline 1.500 & 0,6698002 & $102.109,40$ & $47.317,65$ & $149.427,05$ & 68,07 & 31,55 & 99,62 \\
\hline
\end{tabular}

Hay que tener en cuenta que este contrato de reaseguro de excedentes, para los datos del ejemplo, es equivalente a un reaseguro cuota parte con las siguientes cuotas de retención de la cedente:

$$
k^{\text {seg }}=\frac{M^{\text {seg }}}{S^{\text {seg }}}=\frac{1.000}{2.000}=0,5 \text { y } k^{r e n}=\frac{M^{\text {ren }}}{S^{r e n}}=\frac{150}{200}=0,75
$$

El comportamiento, por tanto, de los capitales de solvencia obligatorios respecto al tamaño del colectivo coincide con los del apartado anterior.

\subsubsection{Reaseguro stop-loss}

En la Tabla 5 se muestran los resutados obtenidos para $\gamma, S C R^{c}, S C R^{r}, S C R^{T o t a l}, S C R_{i}^{c}, S C R_{i}^{r}$ y $S C R_{i}^{\text {Total }}$ de un reaseguro stop-loss con prioridad $M=10.000 €$, para diferentes escenarios de tamaño del colectivo, $n_{0}$. 
Tabla 5. Reaseguro stop-loss con $M=10.000 €$

\begin{tabular}{c|c|c|c|c|c|c|c}
\hline $\boldsymbol{n}_{\mathbf{0}}$ & $\boldsymbol{\gamma}$ & $\boldsymbol{S C R}^{\boldsymbol{c}}$ & $\boldsymbol{S C R}^{\boldsymbol{r}}$ & $\boldsymbol{S C R}^{\text {Total }}$ & $\boldsymbol{S C R}_{\boldsymbol{i}}^{\boldsymbol{c}}$ & $\boldsymbol{S C R}_{\boldsymbol{i}}^{\boldsymbol{r}}$ & $\boldsymbol{S C R}_{\boldsymbol{i}}^{\text {Total }}$ \\
\hline 1 & 1 & 110,86 & 0 & 110,86 & 110,86 & 0 & 110,86 \\
\hline 10 & 1 & $1.089,76$ & 0 & $1.089,76$ & 108,98 & 0 & 108,98 \\
\hline 50 & 0,9858666 & $5.335,31$ & 78,53 & $5.413,83$ & 106,71 & 1,57 & 108,28 \\
\hline 100 & 0,8596754 & $9.296,69$ & $1.531,02$ & $10.827,71$ & 92,67 & 15,31 & 108,28 \\
\hline 500 & 0,3227563 & $17.383,65$ & $36.495,44$ & $53.879,09$ & 34,77 & 72,99 & 107,76 \\
\hline 1.000 & 0,1685419 & $12.150,97$ & $89.519,12$ & $101.670,09$ & 12,15 & 89,52 & 101,67 \\
\hline 1.500 & 0,1125148 & $8.168,70$ & $141.243,20$ & $149.311,90$ & 5,46 & 94,16 & 99,54 \\
\hline
\end{tabular}

Como puede apreciarse en la Figura 6, el valor del parámetro $\gamma$ es inversamente proporcional al tamaño del colectivo salvo para colectivos muy pequeños donde el parámetro $\gamma$ es constante e igual a 1 . Esto es debido a que el reaseguro no interviene en carteras con colectivos muy pequeños, ya que la siniestralidad de la compañía está por debajo de la prioridad $M$. Es lo que sucede para $n_{0}=1$ y $n_{0}=10$, sin embargo, cuando el colectivo empieza a crecer a partir de un determinado umbral, la sinestralidad de la compañía sobrepasa la prioridad y, por tanto, cada vez hay más siniestralidad a cargo del reasegurador, disminuyendo el valor del parámetro $\gamma$.

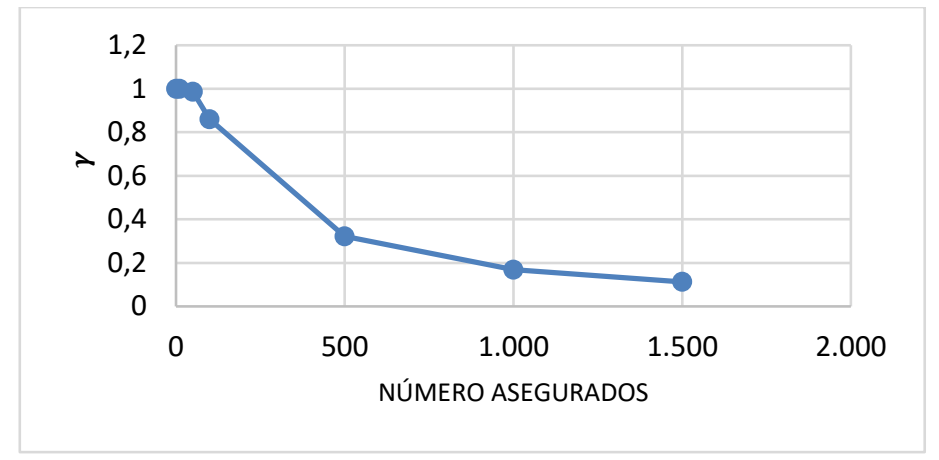

Figura 6: Relación entre $\gamma$ y $n_{0}$

En la Figura 7 se muestra el comportamiento de $S C R^{c}, S C R^{r}$ y $S C R^{\text {Total }}$ con respecto al tamaño del colectivo, $n_{0}$. En el caso del $S C R^{c}$ se aprecian dos tramos diferenciados. En el primero, que se corresponde con tamaños del colectivo pequeños, en este ejemplo hasta 500 asegurados, el $S C R^{c}$ es creciente, esto es debido a que, para colectivos de tamaño muy pequeño, prácticamente toda la siniestralidad de la cartera la asume la cedente por estar ésta por debajo de la prioridad. Conforme va aumentando el tamaño del colectivo, la cedente empieza a compartir el peso de la siniestralidad con el reasegurador disminuyendo el crecimiento del $S C R^{c}$. En el segundo tramo el $S C R_{0}^{c}$ disminuye, ya que cada vez es menor el porcentaje de participación de la cedente en la siniestralidad total de la cartera conforme aumenta el tamaño del colectivo y mayores son los ingresos por primas cobradas, lo que permite a la compañía de seguros disminuir sus dotaciones de $S C R^{c}$. Respecto al comportamiento del $S C R^{r}$, este presenta también dos tramos, en el primero hasta un tamaño de 10 asegurados su valor es cero, debido a que el reasegurador no tiene que dotar $S C R^{r}$ por no superar la siniestralidad de la compañía la prioridad del contrato $M$. En el segundo tramo el $S C R^{r}$ es creciente, ya que cada vez que va aumentado el tamaño del colectivo, mayor es el porcentaje de participación del reasegurador en la siniestralidad total de la cartera. Cabe destacar el comportamiento creciente del $S C R^{\text {Total }}$. 


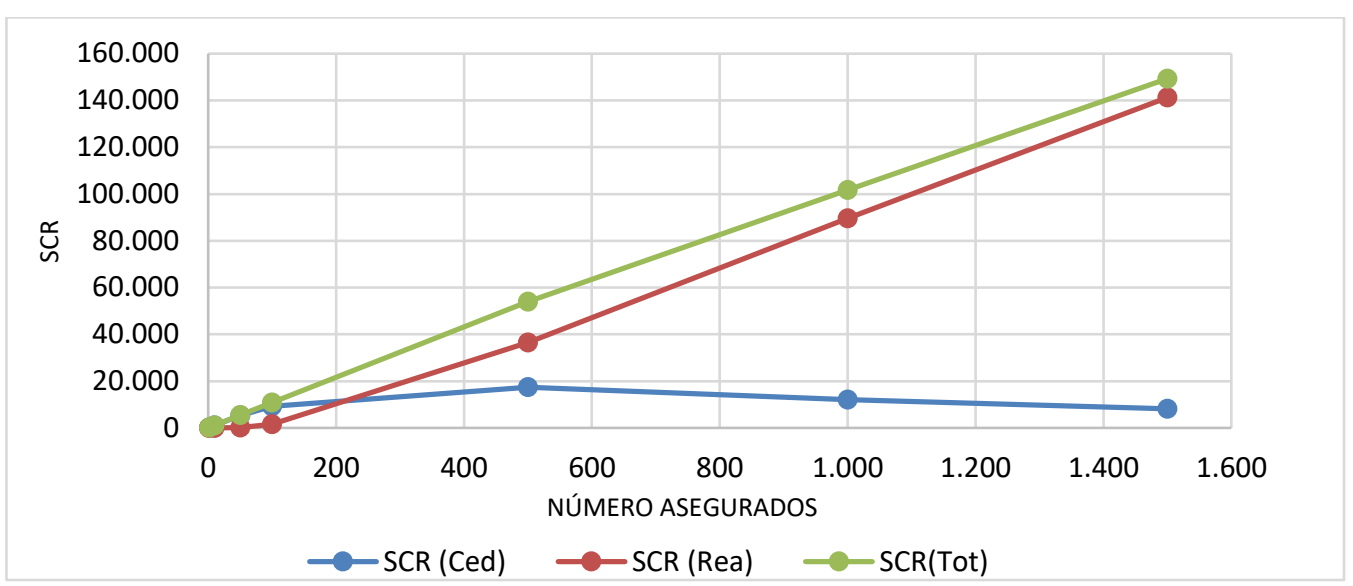

Figura 7: Relación entre $S C R^{c}, S C R^{r}, S C R^{\text {Total }}$ y $n_{0}$

En la Figura 8 se muestra la evolución de $S C R_{i}^{c}, S C R_{i}^{r}$ y $S C R_{i}^{T o t a l}$ respecto al tamaño del colectivo, $n_{0}$. Para este ejemplo, el $S C R_{i}^{c}$ siempre es decreciente y el $S C R_{i}^{r}$ inicialmente es cero y luego siempre es creciente, pero con tasas de crecimiento cada vez menores conforme se acerca a los 1.500 asegurados. Cabe destacar como va disminuyendo el $S C R_{i}^{\text {Total }}$ conforme aumenta el tamaño del colectivo debido a que disminuye el riesgo no sistemático de la cartera.

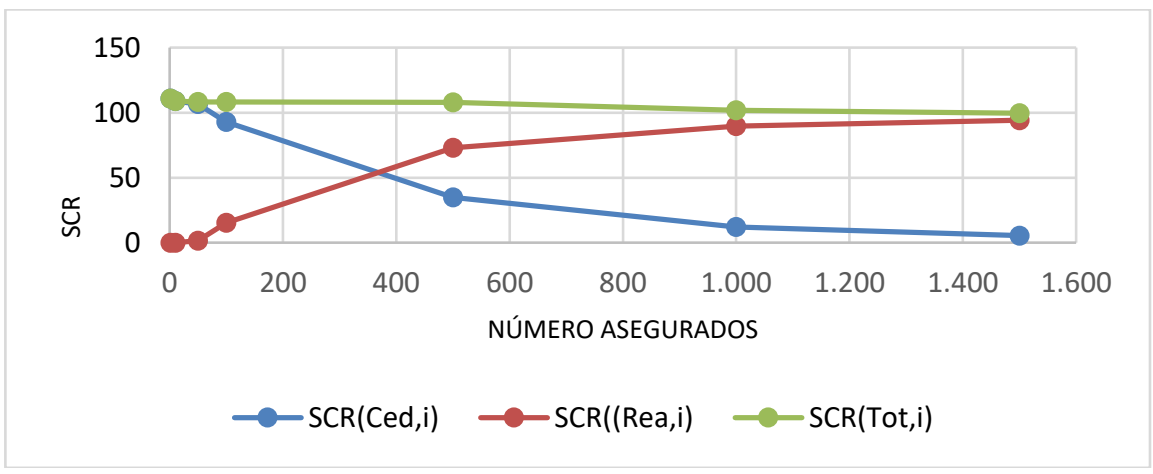

Figura 8: Relación entre $S C R_{i}^{c}, S C R_{i}^{r}, S C R_{i}^{\text {Total }}$ y $n_{0}$

En la Tabla 6 se muestran los resutados obtenidos para $\gamma, S C R^{c}, S C R^{r}, S C R^{\text {Total }}, S C R_{i}^{c}, S C R_{i}^{r}$ y $S C R_{i}^{\text {Total }}$ de un reaseguro stop-loss con prioridad $M=10.000 €$ y con $n_{0}=500$ asegurados, para diferentes edades $x$ del colectivo.

Tabla 6. Reaseguro stop-loss con $n_{0}=500$ y para diferentes edades

\begin{tabular}{c|c|c|c|c|c|c|c}
\hline $\boldsymbol{x}$ & $\boldsymbol{\gamma}$ & $\boldsymbol{S C R}^{\boldsymbol{c}}$ & $\boldsymbol{S C R ^ { \boldsymbol { r } }}$ & $\boldsymbol{S C R ^ { T o t a l }}$ & $\boldsymbol{S C R}_{\boldsymbol{i}}^{\boldsymbol{c}}$ & $\boldsymbol{S C R}_{\boldsymbol{i}}^{\boldsymbol{r}}$ & $\boldsymbol{S C R}_{\boldsymbol{i}}^{\text {Total }}$ \\
\hline 20 & 0,1104973 & $8.160,02$ & $65.549,88$ & $73.709,90$ & 16,32 & 131,10 & 147,42 \\
\hline 40 & 0,1455890 & $10.304,77$ & $60.259,64$ & $70.564,41$ & 20,61 & 120,52 & 141,13 \\
\hline 60 & 0,3227563 & $17.383,65$ & $36.495,44$ & $53.879,09$ & 34,77 & 72,99 & 107,76 \\
\hline 70 & 0,2647072 & $9.844,40$ & $49.578,34$ & $59.422,74$ & 19,69 & 99,16 & 118,85 \\
\hline
\end{tabular}

La Figura 9 muestra el comportamiento del parámetro $\gamma$ respecto a la edad $x$ de los asegurados del colectivo. A mayor edad del colectivo mayor es el porcentaje de siniestralidad de la cedente en la siniestralidad total y por tanto mayor es $\gamma$. Para edades altas esta tendencia se invierte debido al mayor peso que en la operación mixta tiene el seguro inmediato respecto a la renta diferida. A partir de los 70 años, la probabilidad de que el asegurado empiece a cobrar la renta es cada vez más pequeña conforme aumenta la edad, en consecuencia el exceso de siniestralidad creciente derivado del riesgo de fallecimiento lo asume, cada vez más, el reasegurador. 


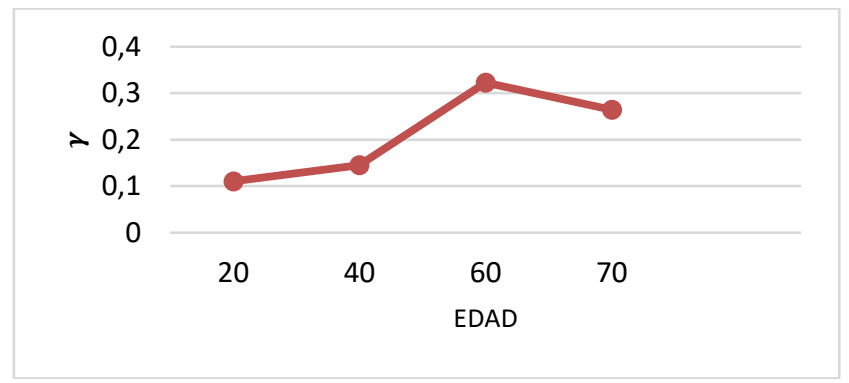

Figura 9: Relación entre $\gamma$ y la edad $x$ de los asegurados

La Figura 10 muestra el comportamiento de $S C R^{c}, S C R^{r}$ y $S C R^{\text {Total }}$ con respecto a la edad $x$ de los asegurados del colectivo. En el caso del $S C R^{c}$ y del $S C R^{r}$ se aprecian dos tramos de edades diferenciados, el primero hasta los 60 años y el segundo a partir de esa edad. Respecto al primer tramo, al ser el parámetro $\gamma$ creciente, el $S C R^{c}$ crece y el $S C R^{r}$ decrece, sin embargo, en el segundo tramo se invierte la tendencia al disminuir el parametro $\gamma$.

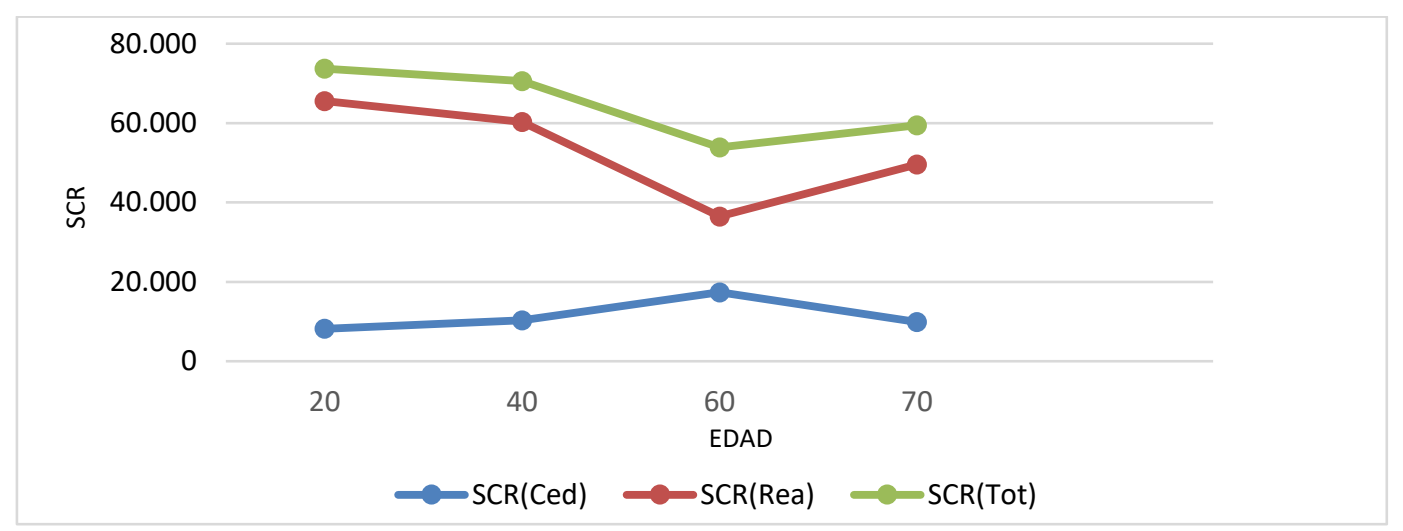

Figura 10: Relación entre $S C R^{c}, S C R^{r}, S C R^{\text {Total }}$ y la edad $x$ de los asegurados

En la Tabla 7 se muestran los resutados obtenidos para $\gamma, S C R^{c}, S C R^{r}, S C R^{T o t a l}, S C R_{i}^{c}, S C R_{i}^{r}$ y $S C R_{i}^{\text {Total }}$ en el reaseguro stop-loss con un tamaño de $n_{0}=500$ asegurados de edad, todos ellos, $x=60$ años y para diferentes valores de la prioridad $M$.

Tabla 7. Reaseguro stop-loss con $n_{0}=500$ y edad de los asegurados $x=60$ años

\begin{tabular}{c|c|c|c|c|c|c|c}
\hline $\boldsymbol{M}$ & $\boldsymbol{\gamma}$ & $\boldsymbol{S C R}^{\boldsymbol{c}}$ & $\boldsymbol{S C R}^{\boldsymbol{r}}$ & $\boldsymbol{S C R}^{\text {Total }}$ & $\boldsymbol{S C R}_{\boldsymbol{i}}^{\boldsymbol{c}}$ & $\boldsymbol{S C R}_{\boldsymbol{i}}^{\boldsymbol{r}}$ & $\boldsymbol{S C R}_{\boldsymbol{i}}^{\text {Total }}$ \\
\hline 5.000 & 0,1672052 & $9.007,02$ & $44.855,73$ & $53.862,75$ & 18,01 & 89,71 & 107,73 \\
\hline 10.000 & 0,3227563 & $17.383,65$ & $36.495,44$ & $53.879,09$ & 34,77 & 72,99 & 107,76 \\
\hline 15.000 & 0,4491538 & $24.172,38$ & $29.689,88$ & $53.862,26$ & 48,34 & 59,38 & 107,72 \\
\hline
\end{tabular}

Cuanto mayor es la prioridad de la cedente, mayor es el peso que asume esta en la siniestralidad total de la cartera en detrimento del reasegurador, esto explica el comportamiento creciente del parámetro $\gamma$ (Figura 11) y del $S C R^{c}$ respecto a la prioridad $M$ y la evolución decreciente del $S C R^{r}$ (Figura 12). 


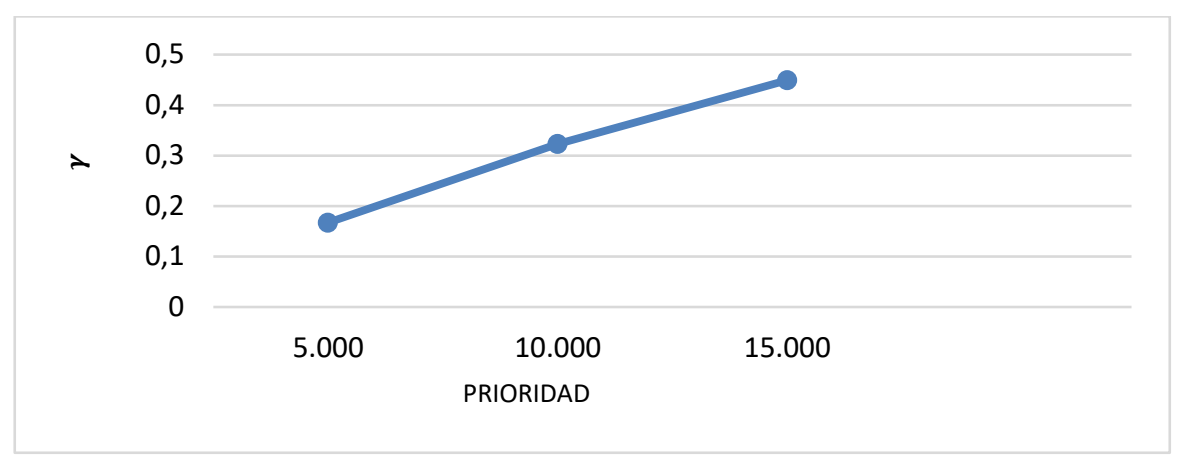

Figura 11:Relación entre $\gamma$ y $M$

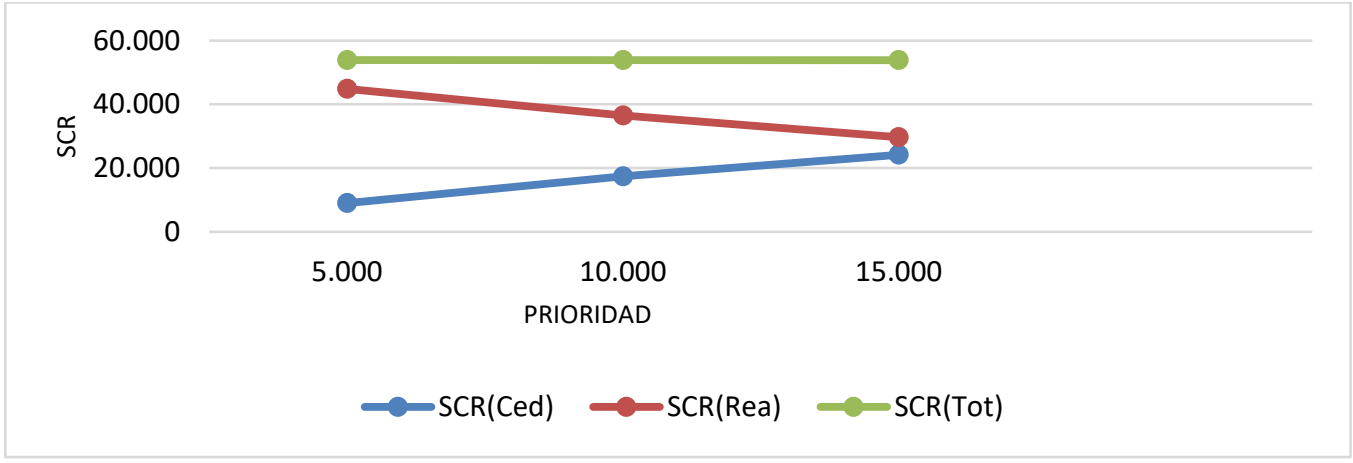

Figura 12: Relación entre $S C R^{c}, S C R^{r}, S C R^{\text {Total }}$ y $M$

\section{Consideraciones finales}

En este trabajo se ha presentado un modelo interno para el cálculo del SCR del riesgo de suscripción de vida, de una cartera que presenta riesgo de mortalidad y riesgo de longevidad. El modelo estándar de Solvencia II propone un sistema de cálculo del $S C R$ modular, basado en la suma correlacionada del capital de solvencia obligatorio de los distintos riesgos que componen la cartera. Sin embargo, esta forma de agregar riesgos conlleva una hipótesis muy restrictiva, ya que asume normalidad de las variables aleatorias marginales que se están considerando y que el coeficiente de correlación entre ellas captura de forma adecuada su dependencia. Sin embargo, en el modelo interno propuesto no se asumen estas hipótesis, ya que la agregación se realiza, para cada simulación, directamente sobre las realizaciones obtenidas por simulación de la variable aleatoria pasivos de la cartera en $t, b_{t}$.

Cuando la cartera presenta riesgo de mortalidad y de longevidad, el $S C R$ total que se obtiene con el modelo estándar recoge un beneficio por diversificación, como consecuencia de asumir coeficientes de correlación negativos entre estos dos riesgos. Sin embargo, en el modelo interno propuesto el beneficio viene explicado fundamentalmente por agregación de asegurados, al disminuir el riesgo no sistemático de la cartera al aumentar su tamaño.

Una vez planteado el modelo interno se ha analizado el efecto mitigador que tienen las distintas modalidades de reaseguro sobre el $S C R$ de la compañía de seguros. Las modalidades de reaseguro analizadas son: el cuota parte, el de excedente y el stop-loss. El cálculo del $S C R^{c}$ y del $S C R^{r}$ pasa por obtener la prima de cada uno de ellos, esta se ha obtenido como una proporción $\gamma$ o $1-\gamma$ respectivamente, de la prima neta de reaseguro de la operación.

En el último apartado hay un ejemplo numérico, estructurado bajo dos supuestos, en el cual se ha calculado el $S C R$ del riesgo de suscrición de vida, para una cartera formada por seguros de vida y rentas de supervivencia, y donde se ha considerado que el colectivo de la cartera es homogéneo en cuanto a edades, sexo y sumas aseguradas. 
En el supuesto 1, sin reaseguro, se ha comparado, para diferentes escenarios con tamaños de colectivos distintos, el $S C R$ que proporciona el modelo interno y el modelo estándar. Se observa que el $S C R$ propuesto por el modelo interno depende del tamaño del colectivo y que su valor, a partir de un determinado número de asegurados, es menor que el que proporciona el modelo estándar.

En el supuesto 2, considerando el reaseguro, se lleva a cabo un análisis de sensibilidad de cómo afecta al $S C R^{c}, S C R^{r}, S C R_{i}^{c}$ y $S C R_{i}^{r}$ la política de reaseguro. En el caso de las modalidades proporcionales de reaseguro, el $S C R^{c}$ es directamente proporcional y el $S C R^{r}$ inversamente proporcional a la cuota de retención de la cedente, de tal manera que cuanto mayor sea esta, es decir, cuanto menos se ceda al reaseguro, mayor será el $S C R^{c}$ y menor el $S C R^{r}$. Esta proporcionalidad se rompe cuando se considera la relación entre el $S C R_{i}^{c}$ y $S C R_{i}^{r}$ con el tamaño del colectivo debido al efecto que el tamaño tiene sobre el riesgo no sistemático del mismo.

En el caso del reaseguro stop-loss cabe destacar el comportamiento creciente y luego decreciente que experimenta el $S C R^{c}$ frente a variaciones en el tamaño del colectivo.

\section{Referencias bibliográficas}

1. Latorre Llorens, L. (2017) Teoría de cópulas. Introducción y aplicaciones a Solvencia II. Cuadernos de la fundación C/219. Fundación Mapfre (2017).

2. Heinen, B. El reaseguro como herramienta de la gestión de capital bajo Solvencia II Europea. Enero 2015. Swiss Re.

3. Dittrich, J. The impact of reinsurance on capital requirements under Solvency II. International Congress of actuaries 7-10. March, 2010. Cape town.

4. Zhou, T. and Kuschel, N. Cost of capital under Solvency II Reinsurance and capital market instruments. Solvency Consulting Knowledge Series. (2012). Munich Re.

5. Pons, M.A y Sarrasí, F.J. Simulación de Monte Carlo aplicada a un modelo interno para calcular el riesgo de mortalidad en Solvencia II. Revista electrónica de comunicaciones y trabajos de Asepuma. Rect@.Volumen 18 (2017), págs. 53-70. DOI 10.24309/recta.2017.18.01.04.

6. Christiansen, M.C. and Niemeyer, A. Fundamental definition of the solvency capital requirement in solvency II. Astin Bulletin, 44, (2014) pages 501-533.

(http://www.journals.cambridge.org/article_S0515036114000105)

7. Fontanals, H. y Ruiz, E. Risc de tipus d'interès. Editorial UOC, 2014. Barcelona.

8. Pons, M.A y Sarrasí, F.J. El reaseguro en el riesgo de mortalidad en Solvencia II. Revista electrónica de comunicaciones y trabajos de Asepuma. Rect@. Volumen 20 - Número 1 (2019), págs. 1-20. DOI 10.24309/recta.2019.20.1.01

9. Minzoni, A. Reaseguro. Editor: Universidad Nacional Autónoma de México. 2009.

\section{Normativa}

- Directiva 2009/138/CE del Parlamento Europeo y del Consejo, de 25 de noviembre de 2009, sobre el seguro de vida, el acceso a la actividad de seguro y de reaseguro y su ejercicio.

- Reglamento Delegado (UE) 2015/35 de la Comisión de 10 de octubre de 2014 por el que se completa la Directiva 2009/138/CE del Parlamento Europeo y del Consejo sobre el acceso a la actividad de seguro y de reaseguro y su ejercicio (Solvencia II).

- UNESPA (2010). Especificaciones técnicas - QIS5, pp. 1-368. Traducción no oficial de las especificaciones técnicas de QIS5.

- EIOPA. Risk-Free Interest Rate Term Structures. https://eiopa.europa.eu/regulationsupervision/insurance/solvency-ii-technical-information/risk-free-interest-rate-term-structures

- Tablas de mortalidad población asegurada española masculina PASEM (2010), BOE núm. 174, de 21 de julio de 2012, pp. 52491 a 52495. 